\title{
Genital psoriasis: update on clinical practices and therapeutic options
}

\author{
Emanuela-Domnica Boieriu, Iulia-Elena Negulet, Ioana-Simona Dinu, \\ Alexandra-Irina Butacu, George-Sorin Tiplica \\ 2nd Department of Dermatology, Colentina Clinical Hospital, \\ "Carol Davila" University of Medicine and Pharmacy, Bucharest, Romania
}

\begin{abstract}
Psoriasis is a chronic skin disease with an important inflammatory compound which associates a worldwide prevalence of $0.5 \%$ to $8.5 \%$. The involvement of the genital region can be associated with both plaque psoriasis and inverse psoriasis and survey-based studies indicate that up to $45.5 \%$ of patients present lesions of genital psoriasis.

Genital psoriasis represents a frequent encounter of psoriasis which is often overlooked and which associates a significant impact on their quality of life, measured by Dermatology Life Quality Index (DLQI) scores which are twice as high as those of psoriatic patients without genital involvement.

Recognising the specific clinical findings represents an important step in establishing the diagnosis and case management. Classical therapeutic options such as topical steroids or modern treatments such as ixekizumab represent important tools in fighting this affliction and regaining the patient's quality of life.

This review aims to emphasize the up-to-date clinical variants of genital psoriasis, the consequences on the quality of life of patients and to present the newest therapeutic options.
\end{abstract}

Keywords: psoriasis, genital psoriasis, quality of life, biological agents

\section{INTRODUCTION AND EPIDEMIOLOGY}

Psoriasis is a chronic skin disease with an important inflammatory compound [1]. The prevalence of the disease in the United States is approximately $2 \%$ to $3 \%$, while worldwide there is a $0.5 \%$ to $8.5 \%$ prevalence [2]. The involvement of the genital region can be associated with both plaque psoriasis and inverse psoriasis [1]. Survey-based studies indicate that up to $45.5 \%$ of patients present lesions of genital psoriasis, while studies that include confirmation of genital lesions through physical examination confirm that $38 \%$ of the patients associate involvement of the genital area, whilst $63 \%$ will develop it at some point in their lifetime [2]. The main risk factors of developing genital psoriasis are male gender, higher severity of the disease evaluated through a higher psoriasis activity score index (PASI) score, onset of the disease over 20 years old, psoriasis lesions of the scalp and nails, inverse psoriasis. There is no link between the articular involvement of psoriasis and the presence of genital lesions. Moreover, body max index and sexual activities are not considered triggering factors for the involvement of the genital area. Another important aspect of the epidemiology of genital psoriasis is that circumcision does not reduce the risk of genital lesions but induces differences in the clinical presentation [1].

\section{AIM AND METHOD}

This review aims to emphasize the up-to-date clinical variants of genital psoriasis, the consequences on the quality of life of patients and to present the newest therapeutic options.

An extensive literature search was conducted in order to accumulate evidence-based data on genital psoriasis. Appropriate literature published between 2016 and 2021 was achived from three com- 
puterised bibliographical databases: PubMed, EMBASE and the Cochrane Library.

The keywords included "psoriasis", "psoriatic" combined with "vulvar", "genital" and "intertriginous". The articles selected were written in English and included case reports, retrospective studies, letters to the editor, randomized clinical trials.

\section{CLINICAL PRESENTATION}

Genital psoriasis remains an underdiagnosed entity as patients rarely disclose this affliction unless questioned. Therefore, a thorough anamnesis and physical examination of the genital area is crucial in establishing the diagnosis [1]. The main triggers of genital lesions are similar to those of non-genital psoriasis, such as stress, drugs, infections, trauma. Moreover, many patients experience worsening of symptoms during and after coitus, most probably being related to friction [3]. One study revealed that the genital involvement of psoriasis is linked to a prolonged duration of the condition and a higher PASI score [4].

The areas that are most frequently involved are the penis shaft and the scrotum, while the glans in men and the labia majora and the perineum in females are less frequently involved [1]. The clinical findings consist of sharply demarcated, red plaques, that lack induration and scale (see figure 1). The specific environment of the genital region including warmth and moisture is responsible for the local maceration and the reduction in scaling. However, scaly lesions can be observed on more keratinized sites of the genital skin [5]. Uncircumcised men commonly present sharply defined plaques localized under the prepuce, while circumcised men present erythematous lesions on the glans [6]. In females, vulvar psoriasis of the labia majora is most often symmetrical, presenting as shiny red plaques without scales in the skin folds or as scaly patches in the outer areas of the labia majora [6].

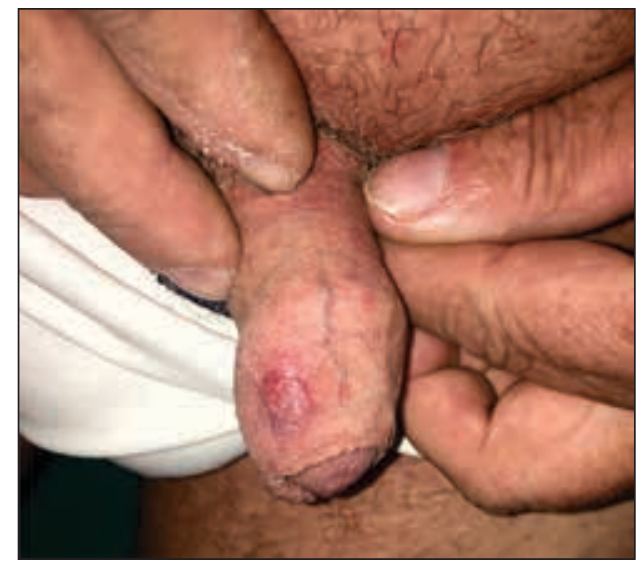

FIGURE 1. Clinical aspect of male genital psoriasis
Particular forms of psoriasis may also involve the genital region, including generalized pustular psoriasis which can associate an onset in the genital region, impetigo herpetiformis, a rare dermatosis of pregnancy which is clinically and histologically consistent with pustular psoriasis or erythrodermic psoriasis which covers more than $90 \%$ of body surface area and may involve the genital region [7].

The PASI score has limitations capturing the involvement of the genital areas [4]. As a result, the Static Physician's Global Assessment of Genitalia was developed for assessing genital psoriasis [2]. Clinical findings such as erythema, induration and scaling are evaluated and scored on a 0-5 scale. The final score is associated with a category of severity (clear, minimal, mild, moderate, severe or very severe). The most important feature of the physical examination is erythema of the genital lesions [2].

The most frequently reported symptom in genital psoriasis is pruritus and is described as an important debilitating symptom by patients [8]. Other reported manifestations include burning sensations and dyspareunia. For women, symptoms associate a higher intensity than men [1]. Men experience decreased sexual function, without erectile disfunction [9]. Gottlieb and al., 2017, developed the Genital Psoriasis Symptoms Scale (GPSS) that serves as a useful tool to measure the intensity of the genital psoriasis symptoms [10]. The GPSS evaluates the following clinical findings: itching, pain, discomfort, stinging, burning, redness, scaling and cracking and each item receives a score from 0 to 10 . Results may range from 0 to 80 . The advantage of the GPSS is that patients feel more comfortable to communicate their symptoms through this type of questionnaire than though a face-to-face conversation with their physician. This tool is applicable to both clinical practices and to clinical trials [10].

\section{THE IMPACT OF GENITAL PSORIASIS ON QUALITY OF LIFE}

Psoriasis has a profound impact on a patient's quality of life, including the psychosocial and sexual well-being. The degree of emotional impact is often disproportionate to the degree of body surface area which is affected [1]. The genital localization of the disease associates an even higher negative influence on the patient's quality of life [6]. 
Patients with genital psoriasis are more frequently depressed than their counterparts without genital involvement, keeping in mind that this category is already more affected than the general population in terms of mental, emotional and social aspects [9].

Furthermore, patients with severe forms of genital psoriasis have a more significant impairment of their quality of life, regardless of the overall disease severity measured through PASI [1].

Additionally, sexual activities are also affected by genital psoriasis [4]. Studies of sexual dysfunction in psoriasis patients found that genital lesions affect more frequently the sexual activities of female patients, even though both genders achieved lower scores compared to healthy cohorts in questionnaires focused on quality of life and sexual health $[1,6]$.

The main reported consequences of genital psoriasis on sexual health included impaired sexual experience during sexual activity, worsening of symptoms after sexual activity, decreased frequency of sexual activity, avoidance of sexual relationships and reduced sexual desire [5]. Patients may also experience pain during sexual intercourse, dyspareunia being more frequently seen in females [9].

Males also experience impairments in sexual function, even though higher scores were achieved in questionnaires measuring quality of life, such as the Dermatology Life Quality Index (DLQI), a skin-generic quality of life questionnaire that includes 10 items to be answered in a 4-point Likert response scale [4].

Several studies reported that DLQI scores were twice as high in patients with genital psoriasis when compared with psoriatic patients without genital involvement [9].

The presence of psoriasis lesions in the genital area was not associated with any statistical increase in erectile dysfunction [1].

Other reported features associated with genital psoriasis included dysuria, insomnia and limitation of common social daily activities [4].

Patients often feel embarrassment and discomfort about discussing these sensitive topics with their healthcare providers. Physicians should take a proactive approach and ask patients afflicted by psoriasis about symptoms involving the genital area $[9,4]$.

The high impact of genital psoriasis on the patient's quality of life represents an important factor in determining appropriate treatment and is considered an independent factor, in addition to the total body surface area of involvement $[4,8]$.

\section{MANAGEMENT OF GENITAL PSORIASIS}

Management of genital psoriasis is challenging due to the sensitive and fragile genital skin which may be prone to a higher risk of local side effects [6].

Topical steroids are recommended as local treatment, especially low-potency topical steroids [1], administrated for a short period of time (2-4 weeks) due to possible adverse reactions [11]. Moderate to strong steroids agents may be beneficial in severe cases [1].

Vitamin D analogues are frequently prescribed in combination with topical steroids, due to their risk to cause irritation in the genital area [6].

Topical calcineurin inhibitors (tacrolimus and pimecrolimus) are efficient for the thinner skin of intertriginous areas [11] and they represent an efficient alternative to topical steroids, being associated with minimal adverse reactions [12].

Antifungal agents may be needed in order to reduce the microbial colonization. They may be used as monotherapy or in combination with low-potency topical steroids [11].

Crisaborole represents a non-steroidal, topical agent, approved for the treatment of mild-to-moderate atopic dermatitis. A recent double-blind, randomized, vehicle-controlled trial demonstrated efficiency and safety of crisaborole $2 \%$ ointment in the treatment of intertriginous, genital, and facial psoriasis [13].

Systemic therapies are recommended in cases of refractory genital psoriasis to topical therapeutic options [14].

Ixekizumab represents a monoclonal antibody against IL-17 used in treatment of moderate-to-severe plaque psoriasis [14]. A randomized, double-blinded, placebo - controlled phase III trial was conducted in 2018 and proved the efficacity of ixekizumab in genital psoriasis [15]. The participants of the study were 149 adults with moderate-to-severe psoriasis that were randomized to receive placebo $(\mathrm{n}=74)$ or ixekizumab $160 \mathrm{mg}$ subcutaneously at week 0 and then $80 \mathrm{mg}$ every 2 weeks following the initial dose $(n=75)$ [15]. 62\% of the patients reported completely resolution of genital erosions, fissures and/or ulcers in 1 week, and $83 \%$ of them in 12 weeks [16]. $60 \%$ of patients reported meaningful improvement in genital pruritus at week 12 and $80 \%$ of patients reported improvement on sexual life, such as the frequency of their sexual activity was never or rarely limited by genital psoriasis [15].

An open label randomized controlled study compared the efficacy and safety of ixekizumab 
versus secukinumab in genital psoriasis. The clinical response appeared in the first two weeks of treatment and was similar between the two drugs. Complete clearance of genital skin was reported at 24 weeks in $68 \%$ patients who received ixekizum$\mathrm{ab}$, and $65 \%$ patients who received secukinumab. An improvement of sexual health of the patients was also reported [17].

\section{CONCLUSIONS}

Genital psoriasis represents a frequent encounter of psoriasis which is often overlooked and

Conflict of interest: none declared

Financial support: none declared

\section{REFERENCES}

1. Kelly A, Ryan C. Genital Psoriasis: Impact on Quality of Life and Treatment Options. Am J Clin Dermatol. 2019 Oct;20(5):639-646.

2. Merola JF, Bleakman AP, Gottlieb AB, Menter A, Naegeli AN, Bissonnette R, Guenther L, Sullivan J, Meeuwis K, See K, Duffin KC. The Static Physician's Global Assessment of Genitalia: A Clinical Outcome Measure for the Severity of Genital Psoriasis. J Drugs Dermatol. 2017 Aug 1;16(8):793-799.

3. Dopytalska K, Sobolewski P, Błaszczak A, Szymańska E, Walecka I. Psoriasis in special localizations. Reumatologia. 2018;56(6):392-398.

4. da Silva N, Augustin M, Langenbruch A, et al. Sex-related impairment and patient needs/benefits in anogenital psoriasis: Difficult-tocommunicate topics and their impact on patient-centred care. PLOS One. 2020;15(7):e0235091.

5. Cather JC, Ryan C, Meeuwis K, Potts Bleakman AJ, Naegeli AN, Edson-Heredia E, Poon JL, Jones C, Wallace AN, Guenther L, Fretzin S. Patients' Perspectives on the Impact of Genital Psoriasis: A Qualitative Study. Dermatol Ther (Heidelb). 2017 Dec;7(4):447-461.

6. Czuczwar P, Stępniak A, Goren A, Wrona W, Paszkowski T, Pawlaczyk M, Piekarska-Myślińska D, Woźniak S, Pietrzak A. Genital psoriasis: a hidden multidisciplinary problem - a review of literature. Ginekol Pol. 2016;87(10):717-721.

7. van der Meijden WI, Boffa MJ, Ter Harmsel WA, Kirtschig G, Lewis FM, Moyal-Barracco M, Tiplica GS, Sherrard J. 2016 European guideline for the management of vulval conditions. J Eur Acad Dermatol Venereol. 2017 Jun;31(6):925-941.

8. Ryan C. Genital psoriasis: the failure of dermatologists to identify genital involvement. Br J Dermatol. 2019 Mar;180(3):460-461.

9. Yang EJ, Beck KM, Sanchez IM, Koo J, Liao W. The impact of genital psoriasis on quality of life: a systematic review. Psoriasis (Auckl). 2018 Aug 28;8:41-47. which associates a significant impact on their quality of life, measured by DLQI scores which are twice as high as those of psoriatic patients without genital involvement.

Recognising the specific clinical findings represents an important step in establishing the diagnosis and case management. Classical therapeutic options such as topical steroids or modern treatments such as ixekizumab represent important tools in fighting this affliction and regaining the patient's quality of life.

10. Gottlieb AB, Kirby B, Ryan C, Naegeli AN, Burge R, Potts Bleakman A, Anatchkova MD, Yosipovitch G. The Development of a PatientReported Outcome Measure for Assessment of Genital Psoriasis Symptoms: The Genital Psoriasis Symptoms Scale (GPSS). Dermatol Ther (Heidelb). 2018 Mar;8(1):45-56.

11. Kivelevitch D, Frieder J, Watson I. Pharmacotherapeutic approaches for treating psoriasis in difficult-to-treat areas. Expert Opin Pharmacother. 2018 Apr;19(6):561-575.

12. Dattola A, Silvestri M, Bennardo L. Update of calcineurin inhibitors to treat inverse psoriasis: A systematic review. Dermatol Ther. 2018 Nov;31(6):e12728.

13. Hashim PW, Chima M, Kim HJ. Crisaborole $2 \%$ ointment for the treatment of intertriginous, anogenital, and facial psoriasis: A double-blind, randomized, vehicle-controlled trial. J Am Acad Dermatol. 2020 Feb;82(2):360-365.

14. Martínez MGL, Martínez-Doménech A, Hernández-Bel P, et al. Successful response of genital psoriasis to ixekizumab: report of six cases. J Eur Acad Dermatol Venereol. 2019 Sep;33(9):e325-e327.

15. Ryan C, Menter A, Guenther L. Efficacy and safety of ixekizumab in a randomized, double-blinded, placebo-controlled phase Illb study of patients with moderate-to-severe genital psoriasis. $\mathrm{Br} \mathrm{J}$ Dermatol. 2018 Oct; 179(4):844-852.

16. Merola JF, Ghislain PD, Dauendorffer JN. Ixekizumab improves secondary lesional signs, pain and sexual health in patients with moderate-to-severe genital psoriasis. J Eur Acad Dermatol Venereol. 2020 Jun;34(6):1257-1262.

17. AlMutairi N, Eassa BI. A Randomized Controlled Ixekizumab Vs Secukinumab Trial to Study the Impact on Sexual Activity in Adult Patients with Genital Psoriasis. Expert Opin Biol Ther. 2021 Feb;21(2):297-298. 